\title{
Skin conductance and arousal in the newborn
}

\author{
G Gladman, M L Chiswick
}

\begin{abstract}
We measured skin conductance continuously from the sole of a foot in babies of different conceptional ages before, during, and for 10 minutes after a 'heel prick' carried out for routine blood sampling. We studied 82 healthy babies whose gestational and postnatal ages ranged from $25-42$ weeks, and 1-73 days.

The median skin conductance level (preheel prick) in babies of $40-43$ weeks' conceptional age was $0.6 \mu \mathrm{S}$ (microsiemens) and differed significantly between awake babies $(1.2 \mu S)$ and those who were asleep $(0.5 \mu S)$. In contrast babies less than $\mathbf{4 0}$ weeks had a significantly lower median skin conductive level $(0.3 \mu \mathrm{S})$ which was identical in awake and asleep babies.

In response to the heel prick all babies became aroused and skin conductance rose sharply and immediately in 21 out of $22(95 \%)$ babies $40-43$ weeks' conceptional age, and in seven out of 23 (30\%) babies 36-39 weeks. The median rise at one minute in babies of $40-43$ weeks was significantly higher than those 36-39 weeks $(2.7 \mu \mathrm{S}$ compared with $0.5 \mu S$ ). No babies less than 36 weeks had a change in their skin conductance after the heel prick. These results are consistent with the notion that 'emotional sweating' is a function of maturity and does not develop until 36 weeks' conceptional age.
\end{abstract}

When a small electrical current at constant voltage flows between two electrodes attached to the skin the amount of current passed is proportional to the skin conductance level. The activity of the eccrine sweat glands, controlled by postganglionic cholinergic fibres of the sympathetic nervous system, is one important factor that influences the skin conductance level. The plantar and palmar sweat glands are activated by emotional arousal. In adults measurements of electrodermal activity from electrodes at these sites have been used by psychologists as a marker of arousal, ${ }^{1}$ by psychiatrists as a biological approach to the study of many psychiatric disorders, ${ }^{2}$ and by criminologists as a marker of deception.

The aims of the present study were to assess whether the resting skin conductance level in babies was influenced by their state of arousal, and to study the effect of a 'heel prick', carried out for the purpose of blood sampling during clinical care, on the skin conductance level.

North Western Regional Perinatal Centre, Neonatal Medical Unit, St Mary's Hospital, Manchester M13 0JH G Gladman

M L Chiswick

Correspondence to: Dr Chiswick.

Accepted 5 June 1990 ultrasound scan; clinical assessment after birth; and radiological bone age (babies on our unit who have a chest radiograph taken also routinely have a radiograph of one lower limb). Gestational age assessed by these methods did not vary by more than two weeks in individual babies, and therefore the mother's dates or early ultrasound assessment was used. We used the expression 'conceptional age' for gestational age plus postnatal age.

Cot nursed babies were lightly clothed under a double layer of blanket and the room air temperature ranged from $21^{\circ} \mathrm{C}$ to $25^{\circ} \mathrm{C}$. Other babies were nursed in incubators at a constant ambient temperature. The rectal and skin temperatures of all babies were within the normal range at the time of study. No baby was visibly sweating at the time of the study and none was receiving anticholinergic drugs or other medications that might interfere with sweating. Measurements of the skin conductance level were timed to correspond with the need for blood sampling by heel prick.

Bipolar silver/silver chloride Beckman electrodes (diameter $=4 \mathrm{~mm}$ ) mounted in plastic cups were attached approximately $2.5 \mathrm{~cm}$ apart to the sole of a foot after cleaning the skin with distilled water and drying it. We used electrolyte medium with a $0.05 \mathrm{M}$ sodium chloride concentration, which was freshly made every week, and we took care to avoid electrolyte seepage from under the electrodes. The electrodes were attached to a skin conductance meter which was constructed for us in the Department of Medical Physics, Central Manchester Health Authority. The equipment provided an alternating current of $10 \mathrm{~Hz}$ at a constant voltage of $100 \mathrm{mV}$, the maximum current permitted to flow between the two skin electrodes being 15 microamperes. Incorporated in the conductance meter, in series with the baby, was a resistor of negligible resistance ( $10 \mathrm{ohms}$ ) compared with the skin. The voltage measured across the series resistor was directly proportional to current flow which, in turn, was directly proportional to the skin conductance level. The meter incorporated a digital display of the skin conductance level within the range $0-20 \mu \mathrm{S}$ (microsiemens), and permanent records were made by pen on a moving paper recorder. The conductance meter was calibrated before each recording session using resistors of known conductances.

After the skin electrodes were attached a 15 minute period of stabilisation was allowed before the 'preheel prick' level of skin conductance was read. We clinically assessed each baby's state of arousal when the reading was made, ascribing an arousal score as follows: $0=$ non-rapid eye movement sleep, no movements, regular breathing; $1=$ rapid eye movement sleep, small movements, irregular breathing; 2='quiet awake', eyes open, slight limb movements; 3='active awake', eyes open, moderate limb movements; and 4=crying and vigorous limb movements. 
The heel prick was carried out on the contralateral foot to which the electrodes were attached and the calf was squeezed eight times to encourage blood flow to the heel. The procedure was carried out by the same person each time (GG). Recordings of the skin conductance level were continued for approximately 10 minutes after the heel prick. Our preliminary studies had shown that when a rise in the skin conductance level was observed after the heel prick the peak value almost always occurred at about one minute after the heel prick (fig 1). Therefore for the purpose of this study we measured the skin conductance level (from the permanent record) one minute after the heel prick and used this in the analysis of the results. The state of arousal was also documented one minute after the heel prick.

The Wilcoxon unpaired test was used to test the significance of comparisons between medians. Permission to study skin conductance in babies had been granted by the local ethics committee and parental consent was obtained in each case.

\section{Results}

Preheel prick skin conductance levels in babies less than $\mathbf{4 0}$ weeks' conceptional age had a relatively narrow range $(0 \cdot 1-0 \cdot 7 \mu S)$ compared with the range in babies of 40 weeks or more among whom individual values were not normally distributed (fig 2). The median preheel prick skin conductance in babies within each conceptional age group less than 40 weeks (28-31 weeks; 32-35 weeks; 36-39 weeks) was identical $(0.3 \mu \mathrm{S})$ and signifi-

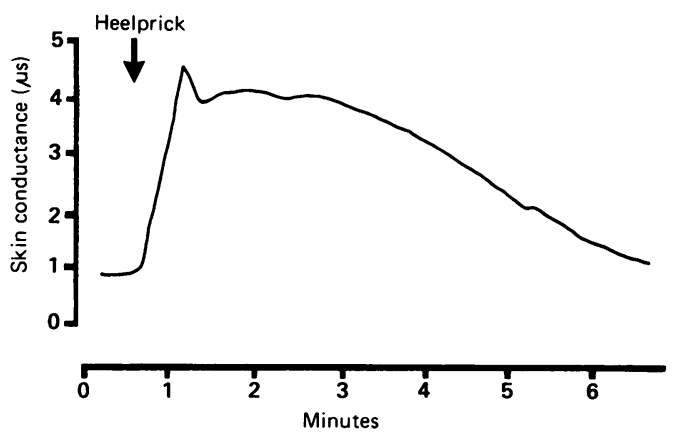

Figure 1 Skin conductance measured from the sole of the foot of a baby in response to a heel prick.

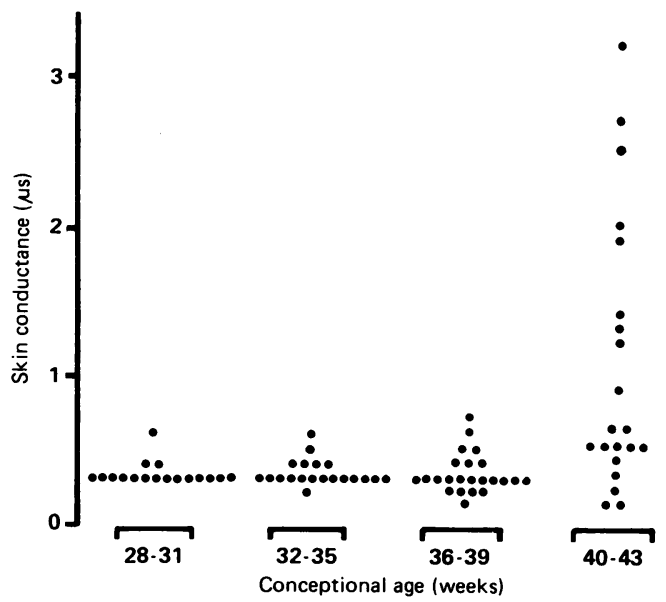

Figure 2 Preheel prick skin conductance levels in babies grouped according to their conceptional ages. Each point represents the result in an individual baby. cantly lower compared with the level in babies $40-43$ weeks $(0.6 \mu S)(p<0.01)$. Within different conceptional age groups there was no significant correlation between the preheel prick skin conductance level and conceptional age, gestational age, or postnatal age.

The distribution of the various states of arousal before stimulation by heel prick was similar in babies of different conceptional age groups (fig 3). Among babies $40-43$ weeks' conceptional age, the preheel prick median skin conductance level was higher in those who were awake (states 2-4) compared with those who were asleep $(0-1)(1 \cdot 2 \mu \mathrm{S}$ compared with $0.5 \mu \mathrm{S}, \mathrm{p}<0.03$ ) (fig 4 ). In contrast, among babies less than $\mathbf{4 0}$ weeks' conceptional age the preheel prick median skin conductance level was identical in awake and asleep babies $(0 \cdot 3$ $\mu \mathrm{S})$.

All babies responded to the heel prick by becoming aroused. The proportion of babies who cried and showed vigorous activity (state 4 ) rose progressively from eight out of $17(47 \%)$ in babies 28-31 weeks' conceptional age to 20 out of 22 (91\%) in those of $40-43$ weeks (fig 3). No baby had a fall in skin conductance level after the heel prick. In all babies less than 36 weeks' conceptional age the skin conductance level after the heel prick did not differ from the resting level. In contrast, in seven out of 23 (30\%) babies 36-39 weeks' conceptional age, and in 21 out of $22(95 \%)$ babies $40-43$ weeks the skin conductance level rose after the heel prick. Among babies 36-39 weeks' conceptional age the proportion who cried vigorously after the heel prick was similar in those who had a rise in their skin conductance level $(71 \%)$ and those who did not show a rise (69\%). Among babies showing a rise in their skin conductance level the median (range) magnitude of the rise was significantly higher in babies $40-41$ weeks' conceptional age $(2 \cdot 7 \mu S(0 \cdot 2-6 \cdot 3 \mu S))$ compared with those 36-39 weeks $(0.5 \mu S(0.1-1.4 \mu S), p=0.005$, fig 5$)$.

Among babies of conceptional ages 36-39 weeks and $40-43$ weeks there was no correlation between the magnitude of the rise in skin conductance level and the resting skin conductance level, conceptional age, postnatal age, initial state of arousal, the state of arousal after heel prick, or the magnitude of the difference between the two states.

\section{Discussion}

Electrodermal activity may be measured as the conductance or the potential difference between two skin electrodes. In the former case it is necessary to pass a current through the skin from an external source (exosomatic) whereas in the latter no external current is required, the potential difference between an active and inactive skin electrode being generated from the skin itself.

We measured skin conductance using equipment that provided an alternating current at a constant voltage. When voltage is held constant the conductance of the circuit between the electrodes varies directly with the current through the skin. The advantages of using a constant voltage as opposed to constant current system, and of using an alternating current source rather than direct current have been reviewed by Venables and Christie. $^{4}$

The mechanisms of electrodermal activity and the factors which influence it have been studied by many investigators. ${ }^{47}$ In our study we measured skin conductance levels, but the physiological 


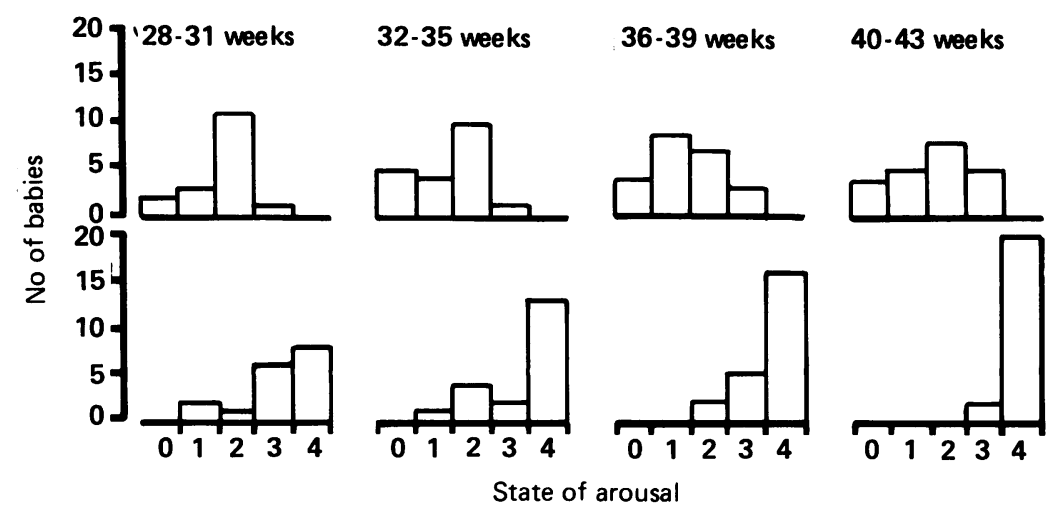

Figure 3 The number of babies, grouped according to conceptional ages, who showed different states of arousal before the heel prick (above) and after (below). State $0=$ non-rapid eye movement sleep, no movements regular breathing; $I=$ rapid eye movement sleep, small movements, irregular breathing; 2 ='quite awake', eyes open, slight limb movements; $3=$ 'active awake', eyes open, moderate limb movements; and $4=$ crying and vigorous limb movements.

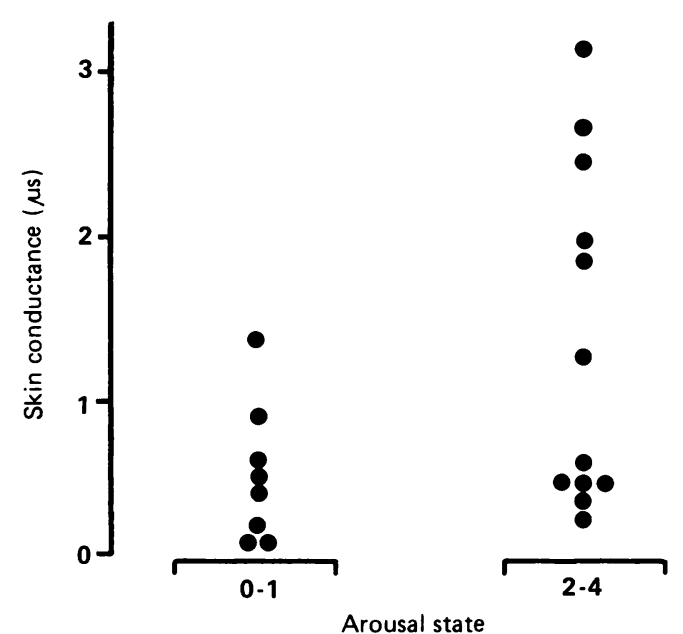

Figure 4 Preheel prick skin conductance levels in babies 40-43 weeks' conceptional age whose states of arousal were $0-1$ compared with those 2-4. Each point represents the result in an individual baby. Comparison of median values: $p=0.03$.

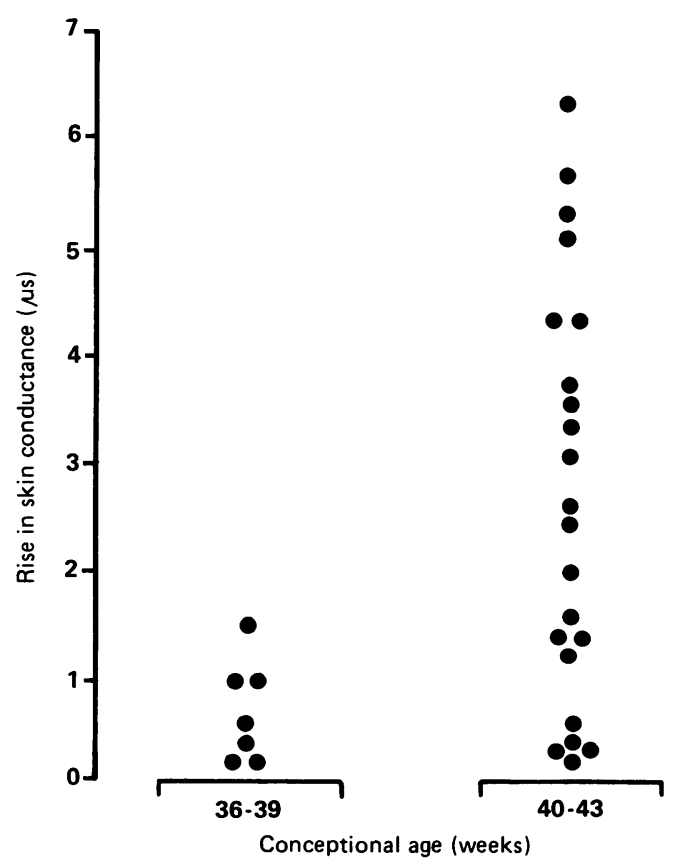

Figure 5 Rise in skin conductance levels from preheel prick levels in babies of 36-39 weeks and 40-43 weeks' conceptional ages. Each point represents the result in an individual baby. Comparison of median values: $p=0.005$. principles are perhaps easier to understand by considering the concept of electrical resistance of the skin, the inverse of conductance. The high resistance to the passage of an electrical current across the skin is a function of the properties of the epidermis. When the sweat ducts become filled, the resistance of the skin is lowered and in this respect each sweat duct behaves like a resistor in parallel. In addition, filling of the ducts is associated with hydration of the stratum corneum, due in part to reabsorption of water from the sweat ducts, and this also contributes to the lowering of skin resistance, the hydrated stratum corneum behaving like a resistor in parallel with the sweat ducts. Skin resistance is also influenced by structures that behave as resistors in series, and these include dry stratum corneum and all tissues beneath it excluding the lumen of the sweat glands and ducts. ${ }^{8}$

We have previously shown that in term babies who are quietly awake evaporative water loss measured from the sole of a foot correlated positively with the skin conductance level measured simultaneously from the other foot ${ }^{9}$. Others have shown that cholinergic blocking drugs cause a fall in skin conductance levels. ${ }^{10}$

The low level of skin conductance we observed in babies less 36 weeks' conceptional age presumably reflects electrical conduction largely through a dry stratum corneum and other epidermal tissues, including the walls but not lumens of the sweat ducts. Muramatsu et al measured skin resistance from the abdominal wall and showed that it was very low (that is, high conductance) at 25 weeks' gestation and rose exponentially (that is, conductance fell) with advancing gestational age. ${ }^{11}$ At first sight these results appear to be at variance with our own but this difference is readily explained. Their measurements largely reflect resistance of the epidermis, especially the stratum corneum, which is very thin in preterm babies and thickens as it matures, resulting in an increase in its electrical resistance. The authors excluded all babies who were visibly sweating and indeed one would not have expected their very preterm babies to have significant sweat gland activity in their abdominal wall skin. As the authors state the added factor of sweat gland activity (which would reduce resistance) was probably negligible. In contrast, our measurements of skin conductance were made from the palm and soles which even in preterm babies are relatively thick. Skin conductance at these sights largely reflects the activity of the sweat glands.

Even when observed to be aroused by a painful stimulus none of our babies less than 36 weeks' conceptional age had a rise in the skin conductance level suggesting that arousal mechanisms and pathways (including peripheral mechanisms) which promote emotional sweating were still immature. Certainly by 28 weeks' gestation babies have a full complement of sweat glands. Although it is well known that the sweat glands are innervated by the sympathetic nervous system, the precise central influences on emotional sweating and skin conductance are not known. Psychophysiologists speak of a 'hierarchy of suprasegmental control' including not only the cerebral cortex but the limbic system, the reticular activating system, and the spinal cord, ${ }^{4}$ but much of the experimental work has been in the context of phasic skin conductance responses rather than tonic levels of skin conductance.

No baby less than 36 weeks' conceptional age 
had a rise in their skin conductance levels after being aroused by a painful stimulus, whereas all except one of the 22 babies of $40-43$ weeks, and seven out of 23 babies of 36-39 weeks showed a rise. While it is true that a smaller proportion of babies less than 36 weeks were observed to cry vigorously after the heel prick this cannot account for failure of their skin conductance to rise because no rise was observed even among those babies who did cry vigorously. Furthermore among babies 36-39 weeks' conceptional age the proportion who cried vigorously after the heel prick was similar in those who had a rise in their skin conductance level $(71 \%)$ and those who did not show a rise $(69 \%)$.

Our results are in keeping with the notion that the skin conductance level rises after an arousal stimulus only in these babies who are mature enough to have developed the capability of emotional sweating and that this first occurs around 36-39 weeks' conceptional age. In support of this, only in babies 40-43 weeks' conceptional age did we observe an association between their state of arousal (awake or asleep) before stimulation and their level of skin conductance. It seems that mature babies can modulate their skin conductance level in accordance with their state of arousal whereas immature babies have a relatively constant skin conductance level regardless of their state.

These observations are consistent with those of Harpin and Rutter who showed that emotional sweating from the palm, measured as evaporative water loss in response to a heel prick, did not develop until 36 weeks after conception. ${ }^{12}$ In the same study these authors observed a positive relationship between palmar water loss and level of arousal which first appeared in infants of 36-37 weeks' conceptional age. In a previous study in which we recorded skin conductance levels for several hours in individual babies we showed that among babies of 37 weeks' conceptional age or more the mean skin conductance level during awake periods was higher than in sleep periods, whereas a significant difference was not seen among less mature babies. ${ }^{9}$

In the present study, among those babies who had a rise in their skin conductance level after arousal by a painful stimulus the magnitude of the rise was significantly higher in babies of $40-43$ weeks' conceptional age than babies 36-39 weeks. This is an important observation suggesting that the onset of emotional sweating is not an all or none phenomenon. It would seem that the less mature babies, after arousal by heel prick, had a fewer number of actively excreting sweat glands, or the volume of sweat per duct was smaller, or hydration of the stratum corneum was less. In terms of the contribution to skin conductance levels it is difficult to distinguish between activity of the sweat glands and hydration of the stratum corneum as the former leads to the latter.

Our study does not address the response of the newborn baby specifically to pain, although pain was used (opportunistically) to stimulate arousal.
Crying and other somatic disturbances indicating arousal occur in response to numerous psychic stimuli as well as to pain. Conversely adults certainly have the ability to suppress somatic manifestations of pain while nonetheless still feeling it. We observed that although immature babies become aroused and may cry in response to a heel prick, they show no rise in their skin conductance level presumably because they are incapable of emotional sweating.

We think it most unlikely that we failed to observe a late rise in skin conductance levels among these babies. Although in this study the skin conductance level was recorded at one minute after the heel prick we continued to observe the level for 10 minutes.

It would appear at first sight that the study of electrodermal activity might have only limited application to the study of pain in immature babies. Although we measured the change of skin conductance levels in response to a painful stimulus we did not measure the 'skin conductance response', which is a different entity used by psychophysiologists to describe a phasic change in skin conductance occurring with a latency of 1 to 2.5 seconds after stimulation, with a time to peak response of less than one second, and an amplitude of 0.01-5.0 $\mu \mathrm{S}$. It was not our aim to study skin conductance responses and our equipment was not designed for this purpose. We cannot rule out the possibility of there being rapid phasic electrodermal activity in response to emotional arousal which has so far gone unnoticed in immature babies and we are exploring this further.

1 Raskin DC. Attention and arousal. In: Prokasy WF, Raskin DC, eds. Electrodermal activity in psychological research. New York: Academic Press, 1973:125-55.

2 Lykken DT. Neuropsychology and psychophysiology in personality research. In: Borgatta EF, Lambert WL, eds. Handbook of personality theory and research. Chicago: Rand McNally, 1968:465-73.

3 Barland GH, Raskin DC. Detection of deception. In: Prokasy WF, Raskin DC, eds. Electrodermal activity in psychological research. New York: Academic Press, 1973:417-77.

4 Venables PH, Christie MJ. Mechanisms, instrumentation, recording techniques and quantification of responses. In: Prokasy WF, Raskin DC, eds. Electrodermal activity in prochological research. New York: Academic Press, 1973:
2-124.

5 Lader MH, Montagu JD. The psycho-galvanic reflex: a pharmacological study of the peripheral mechanism. $f$ Neurol Neurosurg Psychiatry 1962;25:126-33.

6 Fowles DC, Christie MJ, Edelberg R, et al. Publication recommendations for electrodermal measurements. A Committee Report. Psychophysiology 1981;18:232-9.

7 Christie MJ. Electrodermal activity in the 1980's: a review. f $R$ Soc Med 1981;74:616-22.

8 Edleberg $R$. The effects of initial levels of sweat duct filling and skin hydration on electrodermal response amplitude. Psychophysiology 1983;20:550-7.

9 Chiswick ML, Dawson H. Arousal and skin conductance in the newborn. Proceedings of the Physiological Society, 14-15 April 1983. I Physiol (Lond) 1983;342:57-8.

10 Venables PH, Martin I. The relation of palmar sweat gland activity to level of skin potential and conductance. Psychophysiolooy 1967;3:302-11.

11 Muramatsu K, Hirose S, Yukitake K, et al. Relationship between maturation of the skin and electrical skin resistbetween maturation of the skin and
ance. Pediatr Res 1987;21:21-4.

12 Harpin VA, Rutter N. Development of emotional sweating in the newborn infant. Arch Dis Child 1982;57:691-5. 